\title{
Detecting cavitation in mercury exposed to a high-energy pulsed proton beam
}

\author{
Nicholas J. Manzi, a) Parag V. Chitnis, R. Glynn Holt, Ronald A. Roy, and \\ Robin O. Cleveland \\ Department of Mechanical Engineering, Boston University, 110 Cummington Street, Boston, \\ Massachusetts 02215 \\ Bernie Riemer and Mark Wendel \\ Spallation Neutron Source, Oak Ridge National Laboratory, Oak Ridge, Tennessee 37831
}

(Received 5 October 2009; revised 8 February 2010; accepted 10 February 2010)

\begin{abstract}
The Oak Ridge National Laboratory Spallation Neutron Source employs a high-energy pulsed proton beam incident on a mercury target to generate short bursts of neutrons. Absorption of the proton beam produces rapid heating of the mercury, resulting in the formation of acoustic shock waves and the nucleation of cavitation bubbles. The subsequent collapse of these cavitation bubbles promote erosion of the steel target walls. Preliminary measurements using two passive cavitation detectors (megahertz-frequency focused and unfocused piezoelectric transducers) installed in a mercury test target to monitor cavitation generated by proton beams with charges ranging from 0.041 to $4.1 \mu \mathrm{C}$ will be reported on. Cavitation was initially detected for a beam charge of $0.082 \mu \mathrm{C}$ by the presence of an acoustic emission approximately $250 \mu \mathrm{s}$ after arrival of the incident proton beam. This emission was consistent with an inertial cavitation collapse of a bubble with an estimated maximum bubble radius of $0.19 \mathrm{~mm}$, based on collapse time. The peak pressure in the mercury for the initiation of cavitation was predicted to be $0.6 \mathrm{MPa}$. For a beam charge of $0.41 \mu \mathrm{C}$ and higher, the lifetimes of the bubbles exceeded the reverberation time of the chamber $(\sim 300 \mu \mathrm{s})$, and distinct windows of cavitation activity were detected, a phenomenon that likely resulted from the interaction of the reverberation in the chamber and the cavitation bubbles.
\end{abstract}

(C) 2010 Acoustical Society of America. [DOI: 10.1121/1.3353095]

PACS number(s): 43.35.Ei, 43.20.Ye [DLM]

Pages: 2231-2239

\section{INTRODUCTION}

The Spallation Neutron Source (SNS) is an acceleratorbased pulsed neutron source located at the Oak Ridge National Laboratory (ORNL). Currently the SNS is the most intense pulsed neutron beam in the world and is used for scientific research and industrial development. ${ }^{1}$ A proton beam is fired at rate of $60 \mathrm{~Hz}$ into a mercury target. The proton beam is absorbed in a series of collisions (spallation reaction) that release a burst of neutrons and generate heat within the interaction volume. The microsecond time-scale rise in temperature results in an intense acoustic wave propagating throughout the mercury chamber. The expectation is that the amplitude and duration of the tensile portion of the wave are sufficient to produce cavitation bubbles in the mercury. The inertial collapse of bubbles and/or bubble clouds in close proximity to the inner walls of the target vessel may result in mercury jets that impact the boundary, ${ }^{2-5}$ leading to erosion and a reduced service lifetime of the vessel. ${ }^{6}$

The walls of the target vessel in the SNS are made of stainless steel. The photograph in Fig. 1 shows a specimen of the steel wall extracted from the first SNS target module. This specimen is from an interior vessel wall layer that separates the main mercury flow volume from a channel flow that

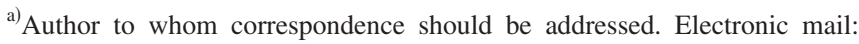
nmanzi@bu.edu
}

is dedicated to cooling the vessel where the proton beam enters the target. The damaged wall is not a containment boundary but the extent of damage is significant especially considering the limited beam power this first target experienced. This target was removed in July 2009 after more than $3000 \mathrm{MW}$ h of operation. However, more than a third of the hours were below $100 \mathrm{~kW}$ and less than $6 \%$ was above 700 $\mathrm{kW}$. At maturity the SNS is designed to operate at $1.4 \mathrm{MW}$ (24 $\mu \mathrm{C}$ charge) although upgrades may allow for even higher power. Aggressive cavitation damage threatens to limit the lifetime and power capacity of the target. A research and development program to develop technologies to mitigate such damage has been underway since 2001, under which the experiments reported in this paper were carried out.

One parameter of interest in assessing the damage potential of cavitation in the SNS is the threshold proton beam energy on target for which the onset of cavitation in mercury occurs. Attempts to externally monitor and characterize cavitation activity have been carried out using a laser Doppler vibrometer (LDV) to measure the vibration induced in the mercury chamber walls by the collapsing bubble clouds. The relationship between the acoustic vibration induced by the impact of the collapsing bubbles and the subsequent pitting damage was used to quantify cavitation-induced damage on the wall. ${ }^{7}$ A threshold for the onset of cavitation activity in mercury was not reported in this reference. 


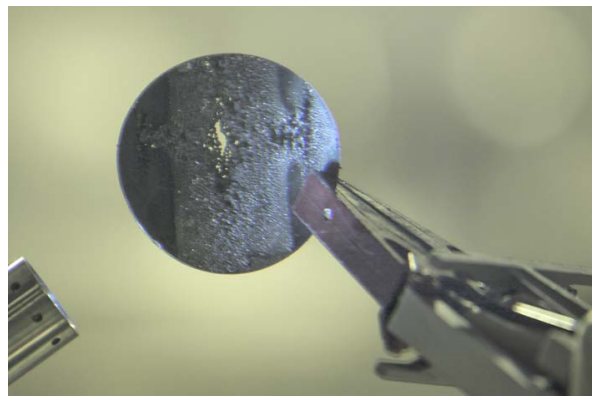

FIG. 1. (Color online) Picture of a specimen extracted from the first SNS target module. This specimen (diameter $\sim 60 \mathrm{~mm}$; original thickness $3 \mathrm{~mm}$ ) is from an interior vessel wall layer that separates the main mercury flow volume from a channel flow that is dedicated to cooling the vessel where the proton beam enters the target.

It is well documented in water that collapsing cavitation bubbles and clouds produce a distinguishable characteristic acoustic emission upon collapse..$^{8-11}$ The goal here was to assess the feasibility of monitoring this acoustic emission to determine a threshold proton beam energy where cavitation first occurs. Megahertz-frequency acoustic transducers immersed in mercury were employed as passive cavitation detectors (PCDs) to record acoustic activity produced within mercury by the collapsing cavitation bubbles.

\section{EXPERIMENTAL SETUP}

\section{A. Mercury target and PCD alignment}

The test target was approximately rectangular in shape with walls made of $4.8-\mathrm{mm}$-thick $316 \mathrm{~L}$ stainless steel. The inner dimensions were $203.2 \times 142.9 \times 41.3 \mathrm{~mm}^{3}$ : A schematic of the target can be seen in Fig. 2. The back wall of the chamber was fitted with two ultrasonic transducers, which were mounted off the axis of the incident proton beam in order to reduce direct radiation exposure to the transducers. The transducers were angled at $11^{\circ}$ so that their acoustic axes

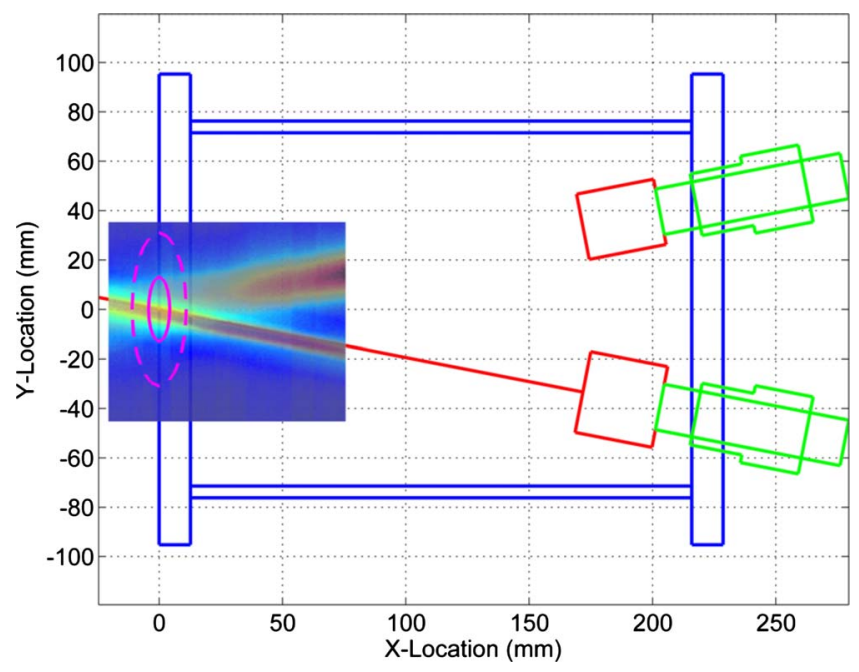

FIG. 2. (Color online) Plan view schematic of the mercury target. The incoming proton beam profiles are shown by the solid ellipse with the width in the $Y$-axis and the height, which is out of the page shown in the $X$-axis. The dashed ellipse shows the large beam profile. The predicted sensing regions for the focused and unfocused transducers are portrayed by the color maps.
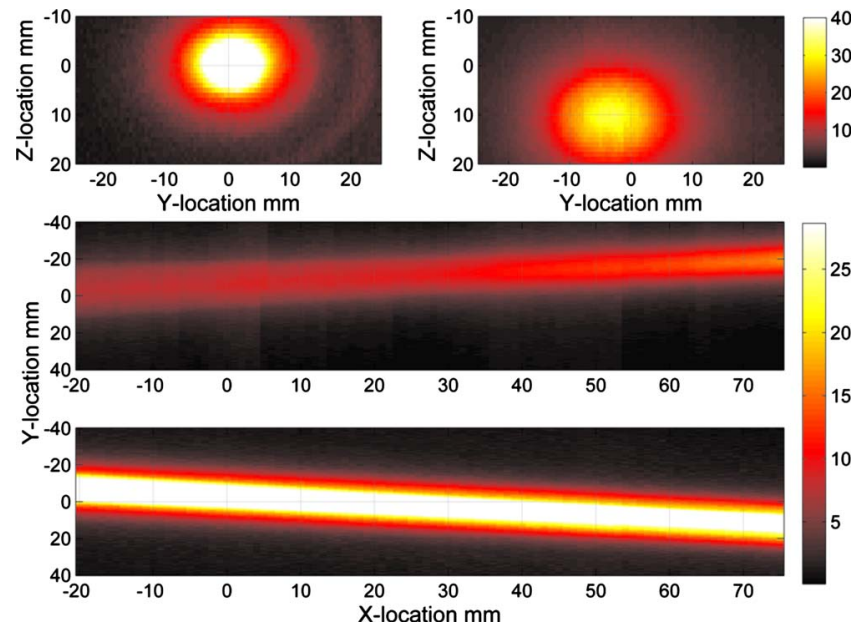

FIG. 3. (Color online) (Top) $Y-Z$ scan in the plane of the incident proton beam for the unfocused $1 \mathrm{MHz}$ focused $2.25 \mathrm{MHz}$ transducers. (Bottom) $X-Y$ scan of the unfocused $1 \mathrm{MHz}$ and focused $2.25 \mathrm{MHz}$ transducers.

crossed where the proton beam entered the chamber. The two transducers employed were a $2.25 \mathrm{MHz}, 25.4 \mathrm{~mm}$ diameter, $200 \mathrm{~mm}$ spherically focused transducer (model V304, Olympus-NDT, Waltham, MA), and a $1 \mathrm{MHz}, 19.05 \mathrm{~mm}$ diameter, unfocused transducer (model V314, OlympusNDT, Waltham, MA). The predicted acoustic fields for the two transducers are also shown in Fig. 2, with the unfocused field calculated using a semi-analytical expression ${ }^{12}$ and the focused source by means of an angular spectrum calculation. Piezoelectric transducers typically act as reciprocal devices, ${ }^{13}$ and therefore field patterns shown in Fig. 2 also correspond to the regions that the transducers are sensitive to when they are used as receivers. The unfocused transducer was chosen to allow cavitation to be detected over a relatively broad region and the focused transducer was chosen to provide a more sensitive response, albeit for a smaller region.

Prior to immersion in mercury the transducers were mounted into the stainless steel wall and their acoustic fields characterized in water. We note that the sound speed in mercury $(\sim 1450 \mathrm{~m} / \mathrm{s})$ is close to that of water $(\sim 1500 \mathrm{~m} / \mathrm{s})$ and therefore the measured spatial acoustic field should be close to that in mercury (the difference in density is only a multiplicative factor). Figure 3 shows results obtained by scanning a broadband point source $175 \mathrm{~mm}$ away from the front face of the transducer in the $Y-Z$ plane. As seen in Fig. 3 the alignment of the transducers' beam axes in the $Z$-axis was approximately $10 \mathrm{~mm}$ off. This suggests a $2^{\circ}-3^{\circ}$ offset in the welded mounting holes used to secure the PCDs. This misalignment was not ideal, but as seen in Fig. 2 the acoustic sensing region of both transducers still coincides with the expected location of the incoming proton beam.

Scans in the $X-Y$ plane were also conducted to check the axial sensitivity of the transducers. As expected the unfocused transducer has a larger diameter beam width, $\approx 20$ $\mathrm{mm}$, than the focused transducer $\approx 6 \mathrm{~mm}$. On the other hand the tighter beam of the focused transducer resulted in great sensitivity at $X=0$, the location where cavitation is anticipated to be most prevalent. The measured beams were well aligned in the $Y$-axis and also in good agreement with the 
predicted profiles shown in Fig. 2. The sensitive regions also extend along the length of the chamber and so cavitation activity will also be detected away from the wall.

The PCD transducer configuration was tested by monitoring cavitation activity produced by a shock wave lithotripter in water. ${ }^{9,14-16}$ The PCDs were mounted in the plate and placed $175 \mathrm{~mm}$ away from the focus of the lithotripter (same distance as in the mercury tests). In this test $50 \mathrm{~m}$ long Bayonet Neill-Concelman (BNC) coaxial cables were employed to mimic the long cable path in the proton beam tests as the electromagnetic pulse generated by the proton beam can adversely affect nearby electronic equipment. Cavitation signals were detected by both PCDs with no spurious signal or noise artifacts induced by the use of $50 \mathrm{~m}$ cables. In one set of mercury experiments the signal level was determined to be too low and an active filter (model 3944, Krohn-Hite, Brockton, MA) with a $10 \mathrm{kHz}$ high pass filter and $20 \mathrm{~dB}$ input gain was placed in a shielded enclosure close to the test target and drove the BNC cable. The data from both PCDs were collected by a digital oscilloscope (LeCroy Waverunner LT344, 8 bits, 500 Msample/s).

\section{B. Pulsed proton beam facility}

The Los Alamos Neutron Science Center (LANSCE) at the Los Alamos National Laboratory (LANL) provided the pulsed proton beam used in the experiment. In the LANSCE facility, a linear accelerator accelerates protons to approximately $84 \%$ of the speed of light $(800 \mathrm{MeV})$. The proton beam was varied from $1 \%$ of full beam charge $(0.041 \mu \mathrm{C})$ to $100 \%$ of full beam charge $(4.1 \mu \mathrm{C})$. A total of 86 shots were fired over a 2-h time period with at least $30 \mathrm{~s}$ in between each shot. The duration of the proton pulse was $0.3 \mu$ s. The spatial distribution of the beam is elliptical with 3:1 $X: Y$ aspect ratio and for the bulk of the experiments reported here a beam of $21 \times 7 \mathrm{~mm}^{2}$ half-width half-height was employed. A full charge of $4.1 \mu \mathrm{C}$ results in $26 \times 10^{12}$ protons with a corresponding flux of 28 $\times 10^{9}$ protons $/ \mathrm{mm}^{2}$ per pulse. Even though the charge is less than in the SNS at $1.4 \mathrm{MW}$ on a per pulse basis the $4.1 \mu \mathrm{C}$ charge is equivalent to the SNS running at $2.1 \mathrm{MW}$ because in the SNS the protons are spread over a larger area. Based on nuclear calculations the absorption of the proton beam by mercury should result in a peak pressure of $35 \mathrm{MPa}$ in the mercury. ${ }^{17}$ To first order the peak pressure scales linearly with proton flux, that is, at $10 \%$ charge $(0.41 \mu \mathrm{C})$ the peak pressure in the mercury will be $3.5 \mathrm{MPa}$. Over the duration of the experiment the temperature of the mercury increased by $7.6 \mathrm{~K}$. The primary objective of the proton beam study was to assess damage to the steel chamber, ${ }^{6}$ and the PCD experiments reported here were subsequently added to the test plan. The digital oscilloscope used to acquire the PCD signals was triggered by a synchronization pulse provided by LANSCE. This trigger was generated by a sensor that detected the proton beam nanoseconds prior to hitting the mercury target and defines time $t=0$ for all waveforms shown below.
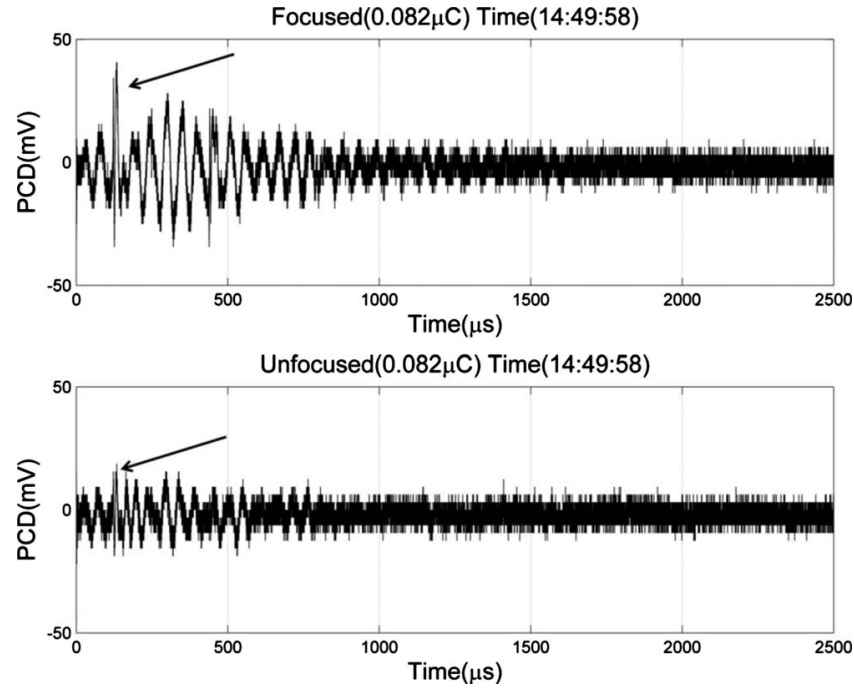

FIG. 4. (Top) Unfiltered signal obtained by the focused transducer for a beam charge of $0.082 \mu \mathrm{C}(2 \%)$. (Bottom) Unfiltered signal obtained by the unfocused transducer for a beam charge of $0.082 \mu \mathrm{C}(2 \%)$.

\section{RESULTS AND DISCUSSION}

Examples of the raw waveforms recorded from both PCDs for a beam charge of $2 \%(0.082 \mu \mathrm{C})$ are shown in Fig. 4. The transducers were located $178.5 \mathrm{~mm}$ from the entrance of the proton beam and therefore (based on the 1450 $\mathrm{m} / \mathrm{s}$ sound speed in mercury) the first arrival associated with acoustic activity where the proton beam enters the mercury will be at $124 \mu \mathrm{s}$. It is expected that the dominant acoustic signals from the thermo-elastic response of the mercury and the dominant cavitation activity will originate close to where the proton beam enters. Signals prior to $124 \mu \mathrm{s}$ can most likely be attributed to acoustic waves generated by the proton pulse elsewhere in the fluid and electronic noise. In the raw data shown in Fig. 4, a low frequency signal $(\approx 20 \mathrm{kHz})$ was observed prior to the expected $124 \mu$ s arrival time. The amplitude of this low frequency signal nearly doubled when the beam charge was increased to 5\%. The low frequency signal became less prevalent with a continuing increase in the beam energy, but was nonetheless still a substantial part of the signal. Simulations of the acoustic field in the chamber (data not shown) indicated that the $\approx 20 \mathrm{kHz}$ signal could be attributed to reverberation in the vertical axis of the chamber; the height of $41.3 \mathrm{~mm}$ has a propagation time of $60 \mu \mathrm{s}$. We note that this signal is 50 times lower than the center frequency of the PCDs and thus has already been reduced by the transfer function of the transducers. Given that emissions associated with cavitation are generally high frequency signals $(\geq 100 \mathrm{kHz})$ a digital high pass filter (fourth order, Butterworth filter with a $100 \mathrm{kHz}$ cut off frequency) was applied to the received data in order to further attenuate these low frequency signals.

Figure 5 shows digitally high passed filtered waveforms collected by the focused and unfocused transducers for beam charges ranging from $1 \%(0.041 \mu \mathrm{C})$ to $100 \%(4.1 \mu \mathrm{C})$. In the waveforms recorded by the focused transducer, for $1 \%$, $2 \%$, and 5\% beam charges, there are signals around 124 and $440 \mu$ s that appear above the noise. The unfocused transducer also picked up weak signals at 124 and $440 \mu$ s with 

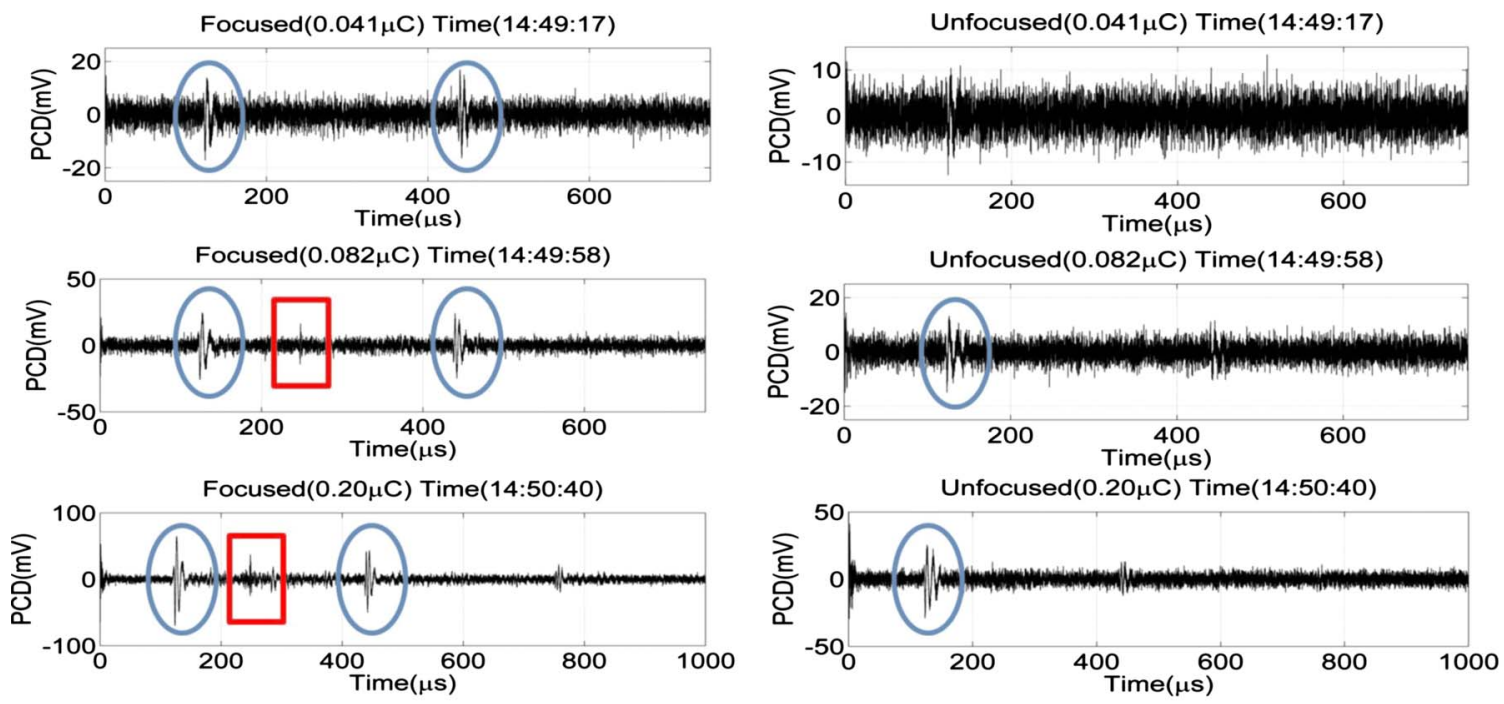

Focused $(0.40 \mu \mathrm{C})$ Time(14:55:03)
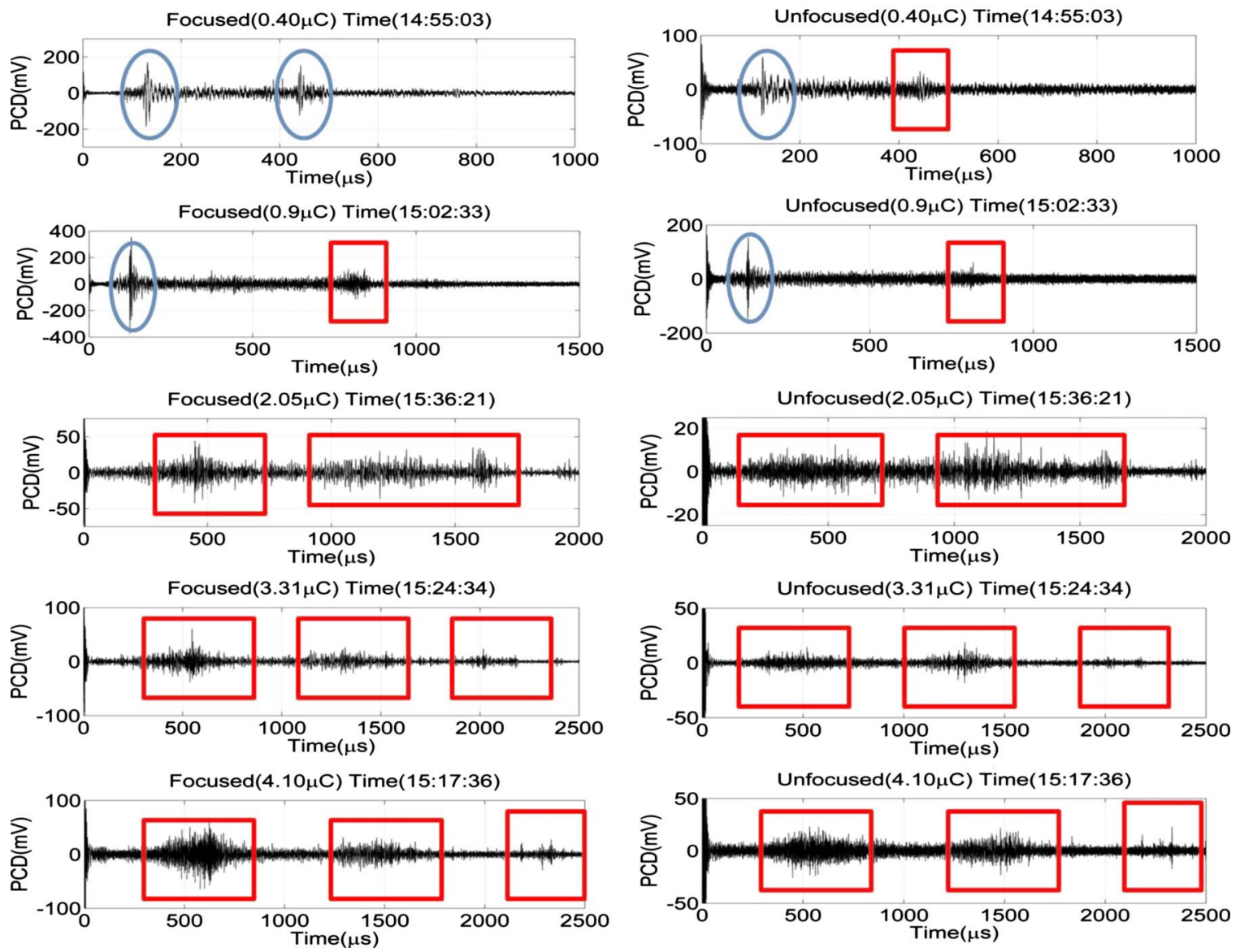

FIG. 5. (Color online) Filtered signals obtained by the focused transducer (left column) and the unfocused transducer (right column) for beam charges of $0.041,0.082,0.20,0.40,0.90,2.05,3.31$, and $4.10 \mu \mathrm{C}$. Ellipses represent acoustic signals and squares represent cavitation signals. Note that the temporal scale increases as charge increases from 0.4 to $2.05 \mu \mathrm{C}$.

the amplitude of this signal approximately half the amplitude produced by the focused transducer. The signal at $124 \mu \mathrm{s}$ corresponds to the first arrival of the initial pressure wave created by rapid heating of the mercury at the entry point of the proton beam (path length approximately $178 \mathrm{~mm}$ ), and it what follows it will be referred to as the "direct wave." The signal at $440 \mu \mathrm{s}$ is caused by a wave that reflects off the back wall, then the front wall, and propagates to the transducer (total path length of about $628 \mathrm{~mm}$ ); this will be re- ferred to as the "reverberation wave." These two signatures are not associated with cavitation activity and will be identified by circles on the figures.

\section{A. Cavitation threshold}

For a beam charge of $1 \%$ there was no evidence of cavitation in either PCD trace. As the proton beam charge was increased to $2 \%(0.082 \mu \mathrm{C})$ the amplitude of the direct 
wave doubled consistent with the expected linear relationship between beam charge and acoustic generation. Evidence of a very weak cavitation emission is present in the focused transducer data at approximately $240 \mu \mathrm{s}$; however, this signal was not detected by the unfocused PCD.

For a beam charge of $5 \%(0.205 \mu \mathrm{C})$ the increase in the amplitude of the direct wave remained consistent with a linear pressure-charge relationship. The focused transducer showed the emergence of two distinct cavitation-related signals: one at $240 \mu \mathrm{s}$ and the other at approximately $290 \mu \mathrm{s}$. These are consistent with the collapse of a cavitation bubble or bubble cloud near the wall where the proton beam was incident. The cavitation event was characterized by a characteristic time $t_{c} \approx 115 \mu \mathrm{s}$, which is the time between the direct wave (that initiates the cavitation) and the acoustic emission from the bubble collapse. ${ }^{10}$ The rebound time $t_{r}$ $\approx 50 \mu$ s is the time between the acoustic emission from the initial bubble collapse and the second emission from the bubble presumably as a result of a rebound. ${ }^{11}$ Again, the cavitation signals were not observed above the noise floor of the unfocused PCD. The focused PCD provides greater sensitivity to cavitation activity and the presence of the cavitation signals indicates that the bubble activity was in or near the sensing volume of the focused PCD. In what follows all transient signals that do not correspond to the direct and reverberation waves will be classified as cavitation signals and in the figures they will be identified by a rectangle.

For a beam charge of $10 \%(0.41 \mu \mathrm{C})$ the arrival of the direct wave was still clearly visible at $124 \mu$ s. However, the rest of the waveform has now changed qualitatively. The expected reverberation wave, which should arrive at $440 \mu \mathrm{s}$, is not distinguishable from a sequence of signals that arrive over the time period of 375-500 $\mu \mathrm{s}$. The spikes appear to grow in amplitude and peak at around $450 \mu$ s and then rapidly decay such that after $500 \mu$ s the signal is barely detectable. The same signal structure was observed in both the focused and unfocused PCDs. We propose that at this beam charge a cloud of cavitation is generated that extends from the entrance window into the bulk of the mercury. These cavitation bubbles are driven hard enough that they have a lifetime greater than the reverberation time of the chamber. As the reverberation wave returns to the front wall it scatters off and/or collapses the cavitation bubbles along its path, which result in the sequence of arrivals observed in the figure with the peak occurring when the reverberation wave interacts with the largest bubbles near the entrance window.

At the next increase in beam charge $(20 \%$ or $0.90 \mu \mathrm{C})$, the focused transducer was still able to pick up the direct wave at $124 \mu \mathrm{s}$. The cavitation activity now extended over a time frame that started almost immediately after the incident pulse arrival and grew with time, reaching a peak at $800 \mu \mathrm{s}$ and then quickly concluding by $900 \mu$ s, with the hint of a rebound signature at $1100 \mu \mathrm{s}$. There is significant "noise" after the emission at $900 \mu$ s suggesting that bubbles remained active for a long time either oscillating and continuing to radiate sound or scattering reverberation waves. The unfocused transducer showed similar behavior but with the direct wave and collapse emission appearing less distinct. These data suggest that cavitation activity is now occurring through much of the mercury. The bubbles further from the window are not driven as hard resulting in a shorter collapse time and a less energetic collapse. In addition, these events are closer to the transducer and therefore will arrive sooner. The cavitation near the window is likely driven the hardest and therefore has the longest time scale, most energetic collapse, and due to the propagation distance will arrive the latest. This is consistent with the strong collapses seen around 800-900 $\mu \mathrm{s}$. The absence of minor peaks for the unfocused transducer may have been because it was sensitive to a much larger region of space and the emissions from the various bubbles did not arrive in phase.

At a beam charge of $50 \%(2.05 \mu \mathrm{C})$ the direct wave at $124 \mu$ s was detectable on both transducers. The focused transducer showed emission activity from 250 to $600 \mu \mathrm{s}$ with a peak around $440 \mu$ s, the time of flight corresponding to the reverberation wave in a bubble free fluid, and may be due to scattering of the reverberation wave from bubbles near the window or acoustic emissions from bubbles that are driven to collapse by the reverberation wave. The cavitation emissions showed a second distinct peak at $1600 \mu$ s after which activity decayed.

At a beam charge of $80 \%(3.31 \mu \mathrm{C})$ no clear direct wave was identifiable but this may be related to the apparent loss of sensitivity of the transducer, as will be described in Sec. III C. The PCDs now recorded three "time windows" of cavitation activity. The first window at $550 \mu$ s does not correspond to the travel time of the reverberated wave; however, the pulse propagated through a two-phase mercury mixture. We speculate that the presence of bubbles in the bulk of the mercury reduced the effective sound speed thus delaying the arrival of the reverberation wave. The second "time window of activity" occurred between 1100 and $1600 \mu$ s similar to the second peak at 50\%. A third distinct event occurred at $2100 \mu$ s suggestive of a rebound.

For proton beam charge of $4.10 \mu \mathrm{C}(100 \%)$ again three windows of cavitation activity were observed. The first window occurred from 400 to $750 \mu$ s with distinct acoustic activity around $600 \mu \mathrm{s}$. This is an additional $100 \mu$ s later than at $80 \%$ beam charge and suggests that the propagation speed of the echo signal may have been further slowed by the presence of bubbles in the mercury. The second distinct "time window" occurred from 1250 to $1600 \mu \mathrm{s}$. A third time window of cavitation signal occurred around $2250 \mu$ s. These windows of cavitation activity were also present in the unfocused signal.

\section{B. Rayleigh collapse}

For the lower beam charges of 0.082 and $0.205 \mu \mathrm{C}$ the PCD recorded the characteristic double bang signature of inertial cavitation with a $t_{C}=120 \mu \mathrm{s}$. This behavior is indicative of single bubble activity or perhaps a coherent cloud at the surface of the wall. If we assume that the emissions are due to a single bubble and further assume that a bubble spends half of this time in a growth phase and half in a collapse phase then $t_{C}$ will be twice the Rayleigh collapse time of a cavity. ${ }^{11,18}$ The maximum radius of the bubble can then be estimated from the Rayleigh collapse time 


$$
R_{\max }=\frac{t_{C}}{1.83} \sqrt{\frac{P_{0}}{\rho_{0}}},
$$

where $P_{0}$ and $\rho_{0}$ are the ambient pressure and density of the mercury. In these experiments the mercury was slightly overpressured and $P_{0}=112 \mathrm{kPa}$ in which case the relationship becomes $R_{\max }=1.6 \mathrm{~m} / \mathrm{s} \cdot t_{C}$ and the measured time of $120 \mu$ s corresponds to a maximum radius of $0.19 \mathrm{~mm}$. For beam charges of $0.41 \mu \mathrm{C}$ and higher the double bang signature was lost and instead there were distinct windows of extended cavitation activity. We speculate that for the higher beam charges the collapse time of the cavitation increased such that it was longer than the reverberation time of the chamber. Based on the reverberation time of the chamber the Rayleigh collapse formula predicts that cavitation bubbles grew to a radius greater than $0.5 \mathrm{~mm}$. It is also likely that at the higher beam charges a cloud of cavitation exists in the mercury. Therefore, when the reverberation wave reflected off the back wall it propagated through the bubbles before they had collapsed inertially. Instead the bubbles were driven to collapse by the reflected acoustic pulse.

\section{Transducer status}

In the course of the experiments, an apparent reduction in the sensitivity of the transducers was observed. This was assessed by monitoring the amplitude of the direct wave during the tests. The pressure amplitude of the direct wave should be linearly proportional to the proton flux and therefore the direct wave amplitude divided by the beam charge should remain constant throughout the experiments. Figure 6 shows the direct wave amplitude normalized by the beam charge as a function of time (top) and the beam charge as a function of time (bottom). Also shown is the amplitude of the noise normalized by the beam charge.

For the first 18 min both the normalized direct wave and the normalized noise remain reasonably constant. At $18 \mathrm{~min}$, when a beam charge of $50 \%(2.05 \mu \mathrm{C})$ was employed, the direct wave amplitude dropped to that of the noise floor. As the beam charge was increased to $4.10 \mu \mathrm{C}$ both normalized levels dropped reaching a minimum at about $35 \mathrm{~min}-$ associated with the last proton beam at $4.10 \mu \mathrm{C}$. In particular, the normalized direct wave amplitude was about 400 times less than the value at $0 \mathrm{~min}$. From 35 to $65 \mathrm{~min}$ the beam charge was stepped down to $0.41 \mu \mathrm{C}$ and the normalized levels started to increase. However, at $65 \mathrm{~min}$, neither the direct wave nor noise level has recovered to their initial values. The data suggest a transient change in the signal levels detected by the PCDs for the higher beam charge.

In order to estimate the performance of the transducers, the response was assessed before, during, and after radiation exposure by transmitting a broadband pulse with the unfocused transducer and sensing the reflection from the window with the focused transducer. This yielded information regarding the combined response of both transducers (plus the reflectivity of the window) rather than the response of an individual transducer. The unfocused transducer was excited with a pulser-receiver [model 5072PR, Panametrics (now Olympus-NDT), Waltham, MA]. Figure 7 shows the evolu-
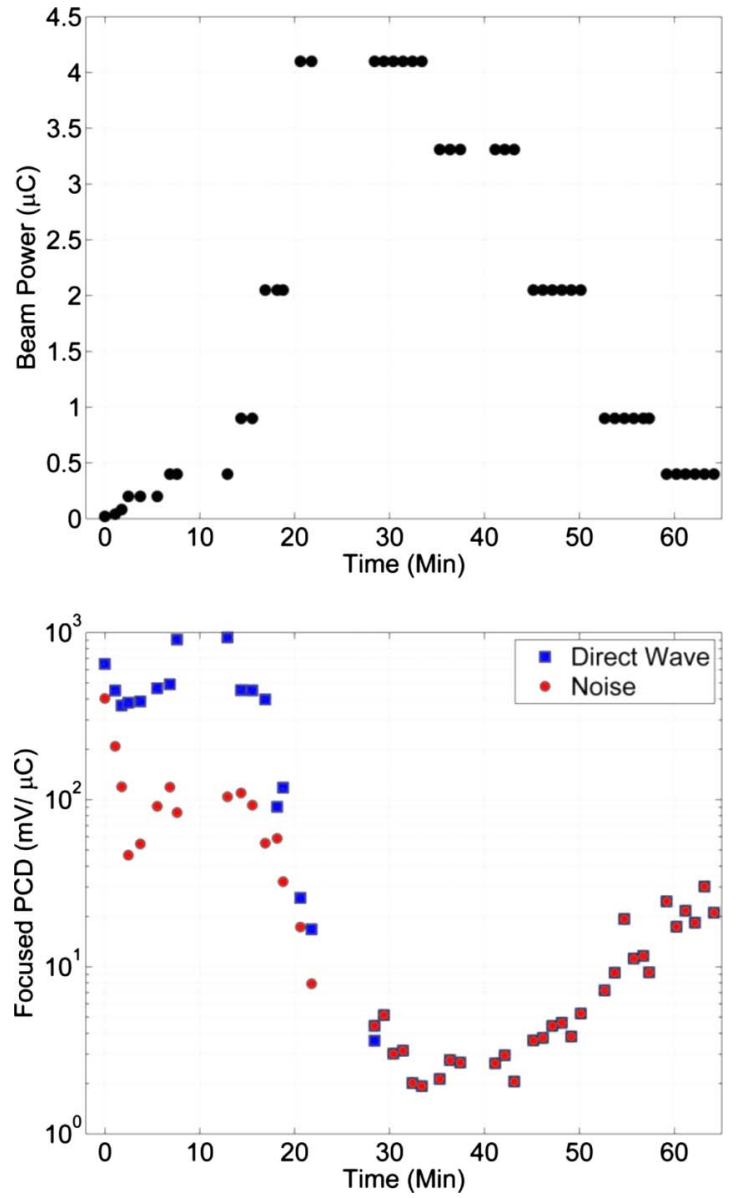

FIG. 6. (Color online) (Top) Response, normalized to beam power, of the focused PCD prior to the arrival of the direct wave (squares) and the subsequent noise floor (circles) of that response as a function of time. (Bottom) Beam charge in microcoloumb as a function of time.

tion of the combined response (temporal and spectral). Prior to exposure to proton beam pulses, the received signal was $400 \mathrm{mV}_{\mathrm{pp}}$ and the spectrum contained two distinct peaks at 0.75 and $1.75 \mathrm{MHz}$. A measurement taken midway in the beam experiments (at a time just after 65 min on Fig. 6, which corresponded to approximately 50 beam pulses) showed a dramatic change in the temporal and spectral responses to the reflected wave. The amplitude dropped by nearly a factor of 50 to about $8 \mathrm{mV}_{\mathrm{pp}}$, and the two peaks at 0.75 and $1.75 \mathrm{MHz}$ are no longer present in the spectrum. The change in amplitude is consistent with the change in the normalized direct wave shown in Fig. 6.

Waveforms taken immediately following the completion of beam exposure show a shape similar to that taken midway through beam exposure; however, the waveforms did show an increase in the signal level to about $70 \mathrm{mV}_{\mathrm{pp}}$, and the spectral signal around $1 \mathrm{MHz}$ is beginning to recover. The transducer's response recovered to near pre-exposure levels (approximately $325 \mathrm{mV}_{\mathrm{pp}}$ ) $20 \mathrm{~h}$ after beam exposure, and was comparable to the pre-beam exposure temporal and spectral responses. It is possible that the proton beam induced a transient change in the piezoelectric efficiency of the transducers, although we would anticipate that in this scenario a permanent depolarization would occur. Another ex- 

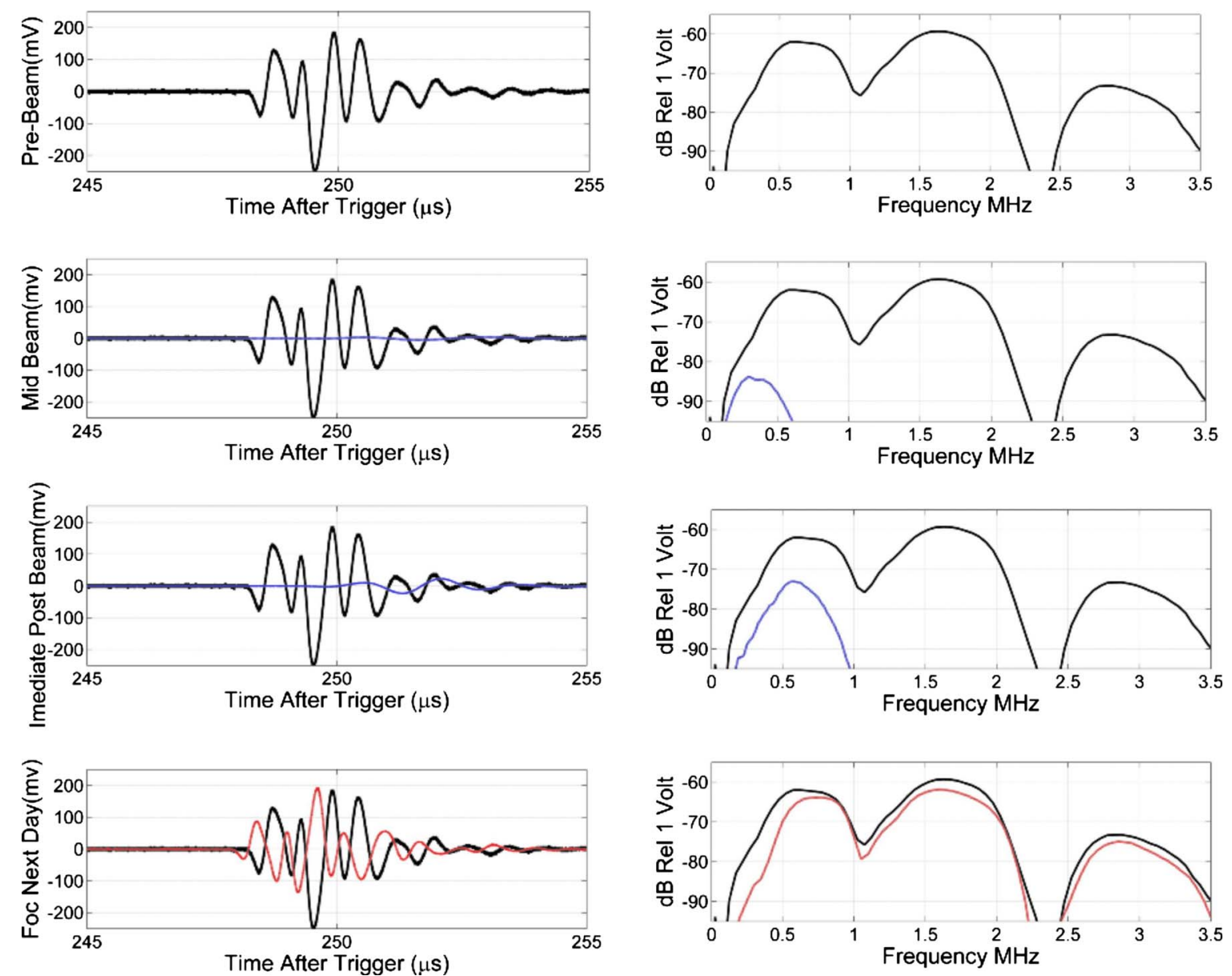

FIG. 7. (Color online) Response of the PCDs prior to, midway through, immediately following, and 1 day after proton beam exposure. (Left) Temporal response measured by pulsing with the unfocused transducer and listening to reflected wave off the front window of the mercury chamber with the focused transducer. (Right) The associated amplitude spectra. For the later responses the initial response is also shown as a solid line.

planation is that the increases in temperature of the bulk fluid may have contributed to the changes. However, only a $7 \mathrm{~K}$ increase in temperature was recorded and this certainly did not affect the sound speed as can be ascertained from the arrival times in Fig. 7. Also the temperature hypothesis is not consistent with the initial recovery seen at the end of the experiments as the temperature should have still be increasing.

A more likely explanation for the change is the presence of bubbles, which attenuated the acoustic wave. The absence of a temporal shift in the arrival time of the pulses suggests that it was probably not due to bubbles in the bulk. Therefore we speculate that the reduction in sensitivity was due to the accumulation of stable bubbles on the face of the transducer. Mercury does not readily wet most solid surfaces (typical contact angles between $130^{\circ}$ and $140^{\circ}$ ) and further our experience indicates that mercury tends to push out particulates and impurities and deposit them on the surfaces of the chamber. Cavitation bubbles formed close to the transducers at high beam charges may have migrated to the surface and remained there-perhaps partially stabilized by the impurities that had been ejected by the mercury. Such a bubble wall would serve to acoustically isolate the sensor. The mercury was slightly overpressured with helium gas and so there was gas in the mercury, which could have diffused into bubbles during cavitation activity. This gas would diffuse back into the mercury in the quiet times between proton pulses. We speculate that as the beam charge was increased the amount of gas driven into the bubbles increased and the bubbles grew to a large enough size or number density that they shielded the PCDs. As the beam charge was reduced the cavitation activity also reduced and so less gas was driven into the bubble and the bubbles shrunk during the quiet periods-this would be consistent with the slow recovery observed in Fig. 6 toward the end of the experiments.

Figure 8 shows PCD measurements after the change in sensitivity of the transducers for a beam charge of $50 \%$. In comparison to the waveforms shown in Fig. 5 for $50 \%$ beam charge it can be seen that the direct wave is no longer detected but three distinct windows of cavitation activity are observed. The first at $450 \mu \mathrm{s}$ is consistent with the emissions shown in Fig. 5. The second at $1600 \mu \mathrm{s}$ is about $100 \mu \mathrm{s}$ later than seen in Fig. 5. A third out-lying distinct cavitation signal also occurred at $1950 \mu \mathrm{s}$, which was not detected in the earlier data. These three windows of cavitation activity are also present in the unfocused signal. These later signals also show an apparent reduction in "noise" signal between the emissions. 
Focused(2.05 $\mu \mathrm{C})$ Time(15:37:21)
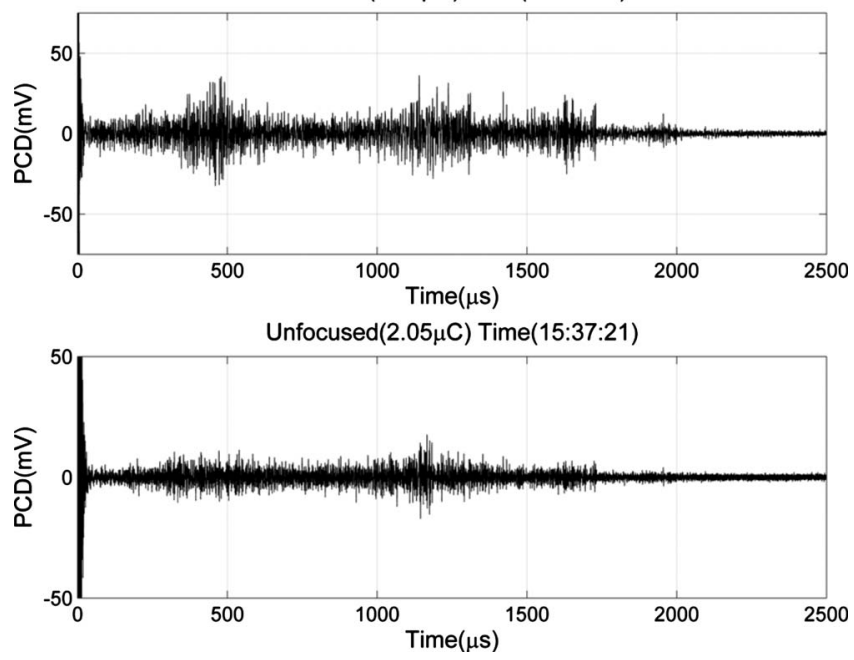

FIG. 8. PCD measurements after the change in sensitivity of the transducers for a beam charge of $2.05 \mu \mathrm{C}(50 \%)$.

\section{Beam shape study}

The previous experiments all employed an elliptical incident beam profile that had a nominal half-width of $21 \mathrm{~mm}$ and a half-height of $7 \mathrm{~mm}$. In order to assess the effect of the spatial profile of the proton beam on cavitation activity, full beam charge experiments were conducted with a small (15 $\left.\times 5 \mathrm{~mm}^{2}\right)$ and a large beam $\left(30 \times 11 \mathrm{~mm}^{2}\right)$. To account for the reduction in sensitivity of both PCDs signals from each transducer were amplified with $20 \mathrm{~dB}$ gain and filtered at 10 $\mathrm{kHz}$ high pass. The digitized signals were then passed through the $100 \mathrm{kHz}$ digital high pass filter described previously. Figure 9 shows representative waveforms taken for the small and large beams.

For the small beam the PCD waveforms look remarkably similar to those for the nominal (medium) beam size in Fig. 5. There are windows of cavitation activity from 400-
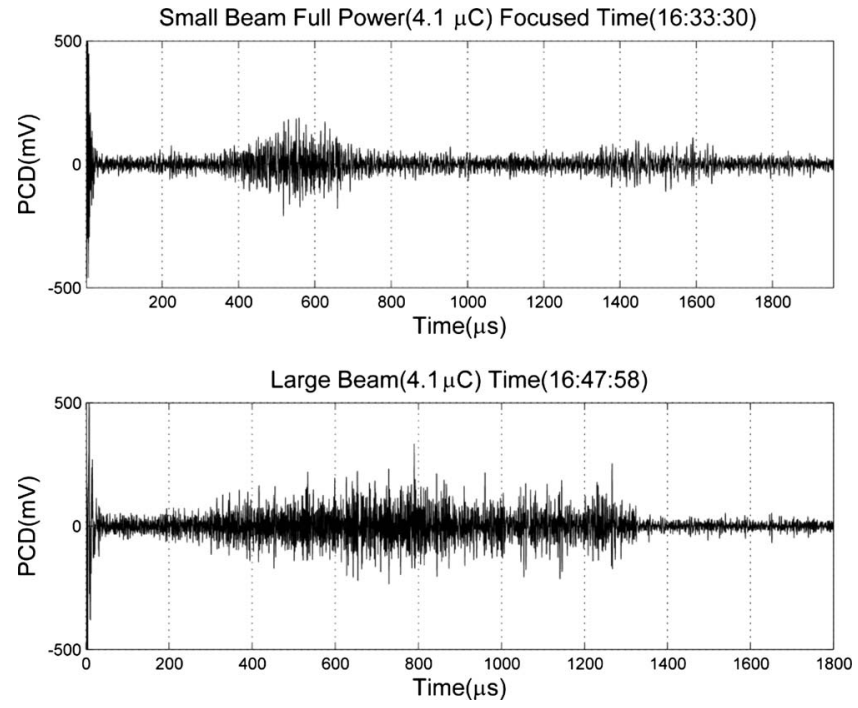

FIG. 9. Representative waveforms obtained for different beam sizes at full charge $4.1 \mu \mathrm{C}$. (Top) Smaller beam size (15 $\mathrm{mm}$ half-width $\times 5 \mathrm{~mm}$ half-height). (Bottom) Larger beam size $(30 \mathrm{~mm}$ half-width $\times 11 \mathrm{~mm}$ half-height).
700 and 1250-1600 $\mu$ s, which are temporally and morphologically similar to what was observed for the regular beam. It is not possible to conclude whether cavitation fields generated by the small and medium beams are significantly different.

In contrast, the waveforms for the large beam showed a distinct change in the cavitation signature. There was no longer the presence of two distinct time windows of cavitation. Instead there was one window of activity, which began slightly earlier $(\approx 300 \mu \mathrm{s})$ and remained present until an abrupt transition at approximately $1300 \mu \mathrm{s}$. This single episode of cavitation suggests that the large beam produced a cavitation cloud of larger spatial extent that interfered so strongly with the acoustic reverberation that the cavitation patterns seen for the medium and small beams did not occur here.

\section{CONCLUSIONS}

We have demonstrated that both focused and unfocused megahertz-frequency immersion transducers, acting as passive cavitation detectors, are able to identify acoustic emissions from cavitation induced by the rapid absorption of a pulsed proton beam in mercury. The focused PCD proved more sensitive and was able to detect cavitation signals for a beam charge as low as $0.082 \mu \mathrm{C}$. Calculations predict a pressure amplitude of $0.6 \mathrm{MPa}$ in the mercury at this charge and this is therefore an estimate of the cavitation threshold in mercury.

PCD measurements taken for a proton beam with a reduced beam size resulted in cavitation signatures similar to the normal-sized beam. However, for a wider proton beam, cavitation signals were only received over one time window indicating that the proton beam energy produced a cavitation field with different spatial and temporal characteristics. Despite exposure to an intense proton beam the two transducers remained operational throughout the process. However, the overall sensitivity and high frequency response of both detectors did decay during exposure. The effect proved transient, as the response recovered the following day, and we speculate that it was due to the buildup of bubbles on the transducer surface.

These results demonstrate that proton beam induced cavitation can be detected in mercury using PCDs. By using a focused detector, one can achieve resolution in both space and time. For low beam powers the cavitation signatures were similar to the double bang emissions expected from inertial cavitation. As the beam power increased it appeared that reverberation in the chamber interacted with the cavitation field to result in more complex cavitation activity with cavitation collapses throughout the mercury. The results suggest that PCDs could be incorporated into the SNS mercury target in order to monitor cavitation activity during operation.

\section{ACKNOWLEDGMENTS}

This work was supported by the ORNL Spallation Neutron Source, which is managed by University of Tennessee (UT)-Battelle, LLC, under Contract No. DE-AC05- 
00OR22725 for the U.S. Department of Energy. The authors would like to thank the LANL and ORNL staffs, in particular, David Felde (ORNL), for technical help with the experiments. This work has benefited from the use of the Los Alamos Neutron Science Center at the Los Alamos National Laboratory, a facility funded by the U.S. Department of Energy.

${ }^{1}$ http://neutrons.ornl.gov/aboutsns/aboutsns.shtml (Last viewed 2/8/2010).

${ }^{2}$ T. Benjamin and A. Ellis, "The collapse of cavitation bubbles and the pressure thereby produced against solid boundaries," Philos. Trans. R. Soc. London, Ser. A 260, 221-240 (1966).

${ }^{3}$ J. Blake and D. Gibson, "Growth and collapse of a vapor cavity near a free surface," J. Fluid Mech. 111, 123-140 (1981).

${ }^{4}$ J. Blake and D. Gibson, "Cavitation bubbles near boundaries," Annu. Rev. Fluid Mech. 19, 99-123 (1987).

${ }^{5}$ T. Leighton, The Acoustic Bubble (Academic, Boston, MA, 1997), pp. 531-545.

${ }^{6}$ J. Haines, B. Riemer, D. Felde, J. Hunn, S. Pawel, and C. Tsai, "Summary of cavitation erosion investigations for the SNS mercury target," J. Nucl. Mater. 343, 58-69 (2005).

${ }^{7}$ M. Futakawa, H. Kogawa, S. Hasegawa, Y. Ikeda, B. Riemer, M. Wendel, J. Haines, G. Bauer, T. Naoe, N. Tanaka, K. Okita, A. Fijiwara, and Y Matsumoto, "Cavitation erosion by proton beam bombarding mercury target for spallation neutron source," Sixth International Symposium on Cavitation, Wageningen, The Netherlands (2006).

${ }^{8}$ A. Vogel and W. Lauterborn, "Acoustic transient generation by laserproduced cavitation bubbles near solid boundaries," J. Acoust. Soc. Am. 84, 719-731 (1988).
${ }^{9}$ A. Coleman, J. Saunders, L. Crum, and M. Dyson, “Acoustic cavitation generated by an extra corporeal shock wave lithotripter," Ultrasound Med. Biol. 13, 69-76 (1987).

${ }^{10}$ A. Coleman, M. Choi, J. Saunders, and T. Leighton, "Acoustic emission and sonoluminescence due to cavitation at the beam focus of an electro hydraulic shock wave lithotripter," Ultrasound Med. Biol. 18, 267-281 (1992).

${ }^{11}$ R. Cleveland, O. Sapozhnikov, M. Bailey, and L. Crum, "A dual passive cavitation detector for localized detection of lithotripsy-induced cavitation in vitro," J. Acoust. Soc. Am. 107, 1745-1758 (2000).

${ }^{12}$ D. Hutchins, H. Mair, P. Puhach, and A. Osei, "Continuous-wave pressure fields of ultrasonic transducers," J. Acoust. Soc. Am. 80, 1-12 (1986).

${ }^{13} \mathrm{M}$. Willatzen, "Ultrasound transducer modeling-General theory and applications to ultrasound reciprocal systems," IEEE Trans. Ultrason. Ferroelectr. Freq. Control 48, 100-12 (2001).

${ }^{14} \mathrm{~L}$. Crum, "Cavitation micro-jets as a contributory mechanism for renal calculi disintegration in ESWL," J. Urol. (Baltimore) 140, 1587-1590 (1988).

${ }^{15}$ G. Delacrétaz, K. Rink, G. Pittomvils, J. P. Lafaut, H. Vandeursen, and R. Boving, "Importance of the implosion of ESWL-induced cavitation bubbles," Ultrasound Med. Biol. 21, 97-103 (1995).

${ }^{16}$ Y. Pishchalnikov, O. Sapozhnikov, M. Bailey, J. Williams, Jr., R. Cleveland, T. Colonius, L. Crum, A. Evan, and J. McAteer, "Cavitation bubble cluster activity in the breakage of kidney stones by lithotripter shock waves," J. Endourol 17, 435-446 (2003).

${ }^{17}$ B. Riemer, "Benchmarking dynamic strain predictions of pulsed mercury spallation target vessels," J. Nucl. Mater. 343, 81-91 (2005).

${ }^{18} \mathrm{P}$. Chitnis and R. Cleveland, "Quantitative measurements of acoustic emissions from cavitation at the surface of a stone in response to a lithotripter shock wave,” J. Acoust. Soc. Am. 119, 1929-1932 (2006). 